\title{
Influence of intraoperative positive end-expiratory pressure level on pulmonary complications in emergency major trauma surgery
}

\author{
Thomas Stueber ${ }^{1}$, Jan Karsten ${ }^{1}$, Nikolas Voigt ${ }^{1}$, Michaela Wilhelmi ${ }^{2}$
}

${ }^{1}$ Department of Anaesthesiology and Intensive Care Medicine, Hannover Medical School, Hannover, Germany

${ }^{2}$ Trauma Department, Hannover Medical School, Hannover, Germany

Submitted: 18 March 2015

Accepted: 28 April 2015

Arch Med Sci 2017; 13, 2: 396-403

DOI: https://doi.org/10.5114/aoms.2016.59868

Copyright @ 2016 Termedia \& Banach

\section{Abstract}

Introduction: Pulmonary complications have a major impact on the morbidity and mortality of critically ill patients with multiple trauma. Intraoperative protective ventilation with low tidal volume may prevent lung injury and infection, whereas the role of positive end-expiratory pressure (PEEP) levels is unclear. The aim of this study was to evaluate the influence of different intraoperative PEEP levels on incidence of pulmonary complications after emergency trauma surgery.

Material and methods: We retrospectively analysed data of multiple trauma patients who underwent emergency surgery within $24 \mathrm{~h}$ after injury in our level I trauma centre $(n=86)$. On the basis of their intraoperative PEEP level, patients were divided into a low PEEP group with a PEEP of $<8 \mathrm{mbar}$ and a high PEEP group with a PEEP of 8 mbar or higher.

Results: Besides differences in body mass index and preoperative oxygenation, there were no differences in patients' baseline data. There was a significant difference between incidence of pneumonia within 7 days after trauma surgery, with an incidence $26.7 \%$ in the low PEEP group and $7.3 \%$ in the high PEEP group $(p=0.02)$. The low PEEP group had higher pulmonary infection scores at days 3 and 5 after surgery. Oxygenation was better in the higher PEEP group postoperatively. There was no difference with respect to the incidence of acute respiratory distress syndrome, the mortality up until hospital discharge or haemodynamic parameters between groups.

Conclusions: Higher PEEP levels were associated with perioperative improvement of oxygenation and a lower incidence of pneumonia, without impairment of haemodynamics. Additional studies should be initiated to confirm these observations.

Key words: intraoperative ventilation, multiple trauma, pneumonia.

\section{Introduction}

Multiple trauma is a major cause of death and disability in the developed and developing world [1]. While early trauma mortality is decreasing, secondary inflammatory organ dysfunction is one of the major issues in trauma critical care [2]. Pulmonary failure arises in up to $20 \%$ of all severely injured patients [3]. Several risk factors such as severity of injury and multiple surgery are associated with pulmonary dysfunction after major trauma [4]. In addition, the quality of mechanical ventilation

\author{
Corresponding author: \\ Dr. Thomas Stueber \\ Department of \\ Anaesthesiology \\ and Intensive Care Medicine \\ Hannover Medical School \\ Carl-Neuberg-Strasse 1 \\ 30625 Hannover, Germany \\ Phone: +49 (0) 5115322292 \\ E-mail: stueber.thomas@ \\ mh-hannover.de
}


during intensive care treatment has an influence on the pulmonary outcome of critically ill patients [5]. Several studies have demonstrated that intraoperative ventilation also influences the incidence of postoperative pulmonary complications [6], especially pneumonia [7] after elective abdominal surgery. High tidal volume ventilation can overstretch the lung and may be associated with subsequent pulmonary injury. The role of intraoperative positive end-expiratory pressure (PEEP) is currently a matter of debate. Higher perioperative PEEP levels may improve oxygenation and reduce the incidence of pneumonia and other pulmonary complications by prevention of cyclic opening and closing of dependent lung areas [8]. Accordingly, ventilation with low PEEP ventilation during surgery was shown to increase mortality in a large database analysis [9]. On the other hand, experimental and clinical data revealed that higher PEEP levels may lead to alveolar overdistension, impair cardiac output and increase pulmonary inflammation [10]. The importance of perioperative mechanical ventilator settings is evolving, but there are insufficient data about which group of patients may or may not benefit from a certain ventilator strategy.

The aim of our current study was to evaluate the association of different intraoperative PEEP levels with the incidence of pneumonia and other postoperative pulmonary complications in the acute perioperative phase of severely injured patients.

\section{Material and methods}

\section{Study design}

The Ethics Committee of the Hannover Medical School approved the study protocol and waived the need for ethical approval due to the retrospective design and anonymization of data (No. 1867-2013). We retrospectively analysed the electronic records of all severely injured patients admitted to our level I trauma centre between June 2010 and November 2013. We included all adult multiply injured patients who underwent emergency surgery within the first $24 \mathrm{~h}$ after injury that had a duration of surgery of more than 90 min in our analysis. Multiple injury was defined by an Injury Severity Score (ISS) $>16$ with trauma to more than 1 body region. We excluded patients with an ISS $<16$ or with a severe injury (ISS > 16) to only one body region. Furthermore, we excluded multiple trauma patients who had no surgery within $24 \mathrm{~h}$ after injury, who had short procedures with an operating time shorter than 90 min or who had surgery outside our trauma centre and were secondarily transferred to our centre. Patients with missing data regarding their body height, intraoperative anaesthetic manage- ment and/or postoperative intensive care management were also excluded.

\section{Patients' characteristics}

For characterisation and comparative purposes of the study population we assessed known risk factors for pulmonary complications, such as gender, age, GCS at scene, base excess on admission, ISS and Abbreviated Injury Scale (AIS) score for the chest region. Furthermore, we calculated the Lung Organ Failure Score (LOFS) for each patient. This score predicts severe pulmonary complications in multiple trauma patients including chest trauma and was calculated from the Trauma Registry data (German Trauma Society) of more than 30000 patients [4].

\section{Intraoperative data}

Intraoperatively collected data included: duration of emergency surgery (minutes), transfusion of packed red blood cells and fresh frozen plasma (units), infused crystalloid and colloid volume $(\mathrm{ml})$, vasopressor use, sedation (total intravenous or balanced anaesthesia), tidal volume $(\mathrm{ml})$, respiratory frequency (breaths per minute), peak airway pressure and PEEP level (mbar). On the basis of intraoperative PEEP level, patients were divided into a low PEEP group with a PEEP of $<8$ mbar and a high PEEP group with a PEEP of 8 mbar or higher. The PEEP cut-off was determined before data acquisition and analysis.

\section{Postoperative data}

Postoperatively assessed data comprised ventilator settings, e.g. PEEP, tidal volume, plateau pressure, respiratory rate and fraction of inspired oxygen $\left(\mathrm{FiO}_{2}\right)$ on postoperative day (POD) 1, 3 and 5 after intensive care unit (ICU) admission at 12:00 a.m.

\section{Outcome}

Our primary outcome of interest was the incidence of clinically diagnosed pneumonia within 7 days after emergency surgery according to criteria as described by Johanson [11].

Pneumonia was clinically suspected upon the presence of new or progressive pulmonary infiltrates on chest radiographs plus two or more of the following criteria: 1) fever $\geq 38.5^{\circ} \mathrm{C}$ or hypothermia $<36^{\circ} \mathrm{C}$, 2) leukocytosis $\geq 12000 \mathrm{WBC} /$ $\mathrm{mm}^{3}$ or leukopenia $<4000 \mathrm{WBC} / \mathrm{mm}^{3}$, 3) purulent sputum, 4) worsening gas exchange.

We calculated the modified Clinical Pulmonary Infection Score (CPIS) as described by Pelosi et al. (mCPIS) from clinically available data on POD 1 , 3 and 5 after emergency surgery [12,13]. Furthermore, the incidence of acute respiratory distress 
Assessed for eligibility $(n=382)$

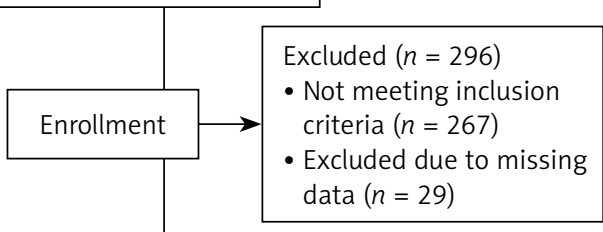

Pre-determinated allocation

$+$

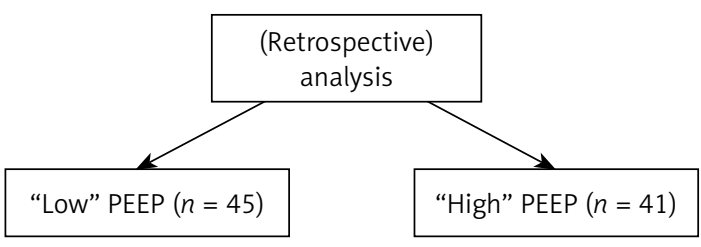

Figure 1. Flow diagram

syndrome according to the Berlin definition and the mortality up until discharge from hospital were documented. We calculated the Horowitz indices (PF ratio) from the first blood gas analysis after arrival at the ICU and on POD 1, 3 and 5 at 12:00 a.m. after surgery as a parameter for oxygenation. We assessed need for vasopressors, amount of fluid therapy, perioperative lactate levels and postoperative creatinine levels as surrogate parameters for possible impairment of circulation by higher PEEP levels.

\section{Statistical analysis}

Variables related to patients' characteristics, intra- and postoperative data and outcome were reported. All continuous data are expressed as means \pm SD unless stated otherwise. Variance analysis by repeated measures procedures (Greenhouse-Geisser $\varepsilon$ method) were used for statistical analysis. Interactions between groups were tested by the Mann-Whitney $U$ test, Kruskal-Wallis test and $\chi^{2}$ test. $P$-value $<0.05$ was considered as statistically significant (two-sided). Statistics were carried out using the IBM SPSS Statistics software tool (Version 21.0, IBM Corp., Armonk, NY, USA).

\section{Results}

A total of 382 patients were screened for this study. Out of these, 86 patients met the inclusion criteria and entered the final analysis (Figure 1). There were no significant differences concerning patients' characteristics between groups besides a significant difference in body weight and baseline oxygenation (Table I). Most patients underwent endotracheal intubation in a pre-clinical setting (70.6\%). There was no significant difference in pre-clinical intubation rate between study groups (Table I). All patients received perioperative antibiotic prophylaxis with a $2^{\text {nd }}$ generation cephalosporin (in the case of a known allergy, clindamycin) according to a local standard operating procedure.

During general anaesthesia for emergency surgery, aside from airway pressure settings due to different PEEP groups (low PEEP vs. high PEEP), there were no significant differences in duration of surgery, parameters of fluid resuscitation and volume management, vasopressor use, maintenance of anaesthesia or tidal volume (Table II). There was a significant difference in tidal volume per kg of predicted body weight intraoperatively. Tidal volume per $\mathrm{kg}$ of predicted body weight was $8.4 \pm 1.2 \mathrm{ml}$ in the

Table I. Patients' characteristics on admission to emergency department ( $<24 \mathrm{~h}$ before surgery)

\begin{tabular}{|c|c|c|c|}
\hline Parameter & $\begin{array}{l}\text { Low PEEP group } \\
\quad(n=45)\end{array}$ & $\begin{array}{l}\text { High PEEP group } \\
\quad(n=41)\end{array}$ & $P$-value \\
\hline Sex (male/female) & $34 / 11$ & $34 / 7$ & 0.40 \\
\hline Age [years] & $42.8 \pm 18.7$ & $38.1 \pm 16.5$ & 0.22 \\
\hline $\mathrm{BMI}\left[\mathrm{kg} / \mathrm{m}^{2}\right]$ & $25 \pm 4$ & $29 \pm 7$ & 0.04 \\
\hline Base excess [mmol/l] & $-4.0 \pm 4.2$ & $-3.6 \pm 4.0$ & 0.78 \\
\hline Lactate [mmol/l] & $2.7 \pm 2.2$ & $3.0 \pm 1.9$ & 0.29 \\
\hline Creatinine $[\mu \mathrm{mol} / \mathrm{l}]$ & $90.7 \pm 32.4$ & $93.2 \pm 33.6$ & 0.77 \\
\hline LOFS & $16 \pm 3$ & $17 \pm 3$ & 0.41 \\
\hline ISS & $27 \pm 9$ & $29 \pm 9$ & 0.26 \\
\hline AIS $_{\text {Thorax }}$ & $3 \pm 1$ & $3 \pm 1$ & 0.38 \\
\hline GCS & $11 \pm 4$ & $11 \pm 5$ & 0.97 \\
\hline Pre-clinical intubation (\%) & 61.4 & 80.5 & 0.55 \\
\hline
\end{tabular}

Data expressed as mean \pm SD. BMI - body mass index, LOFS - lung organ failure score, ISS - injury severity score, AIS - abbreviated injury score, GCS - Glasgow Coma Scale at scene, low PEEP group: < 8 mbar, high PEEP group: $\geq 8$ mbar. 
low PEEP group and $7.8 \pm 0.9 \mathrm{ml}$ in the high PEEP group. Postoperative respiratory data assessed as median values calculated from parameters on POD 1,3 and 5 after ICU admission revealed no significant differences except airway pressures (PIP, PEEP) between groups as well (Table III).

Postoperative respiratory status and other outcome parameters are reported in Table IV. Comparing the incidence of early onset pneumonia within the first 7 days after initiation of mechanical ventilation within the low and the high PEEP group, we found a significant differences (26.7\% vs. $7.3 \%$; $p<$ 0.05). On POD 3 and 5, the mCPIS were significantly lower in the high PEEP group (POD 3: $1 \pm 1$; POD 5: $2 \pm 1$ ) as compared to the low PEEP group (POD 3: $1 \pm 1$ vs. $3 \pm 2, p \leq 0.001$; POD $5: 2 \pm 1$ vs. $3 \pm 2$, $p=0.006$ ) (Table IV). There were no differences in postoperative lactate or creatinine levels.

Oxygenation as expressed by the PF ratio was significantly better in the high PEEP group prior to admission to the intensive care unit (postoperative PF ratio [mm Hg]: $414 \pm 136$ vs. $345 \pm 124 ; p=$ 0.02). On POD 1, 3 and 5 no significant differences could be identified for PF ratio between groups and the incidence of acute respiratory distress syndrome. Early mortality during hospital stay did not differ between groups either (Table IV).

\section{Discussion}

In this retrospective analysis higher intraoperative PEEP levels of 8 mbar and higher were associated with significantly improved oxygenation after

Table II. Intraoperative data

\begin{tabular}{|lccc|}
\hline Parameter & $\begin{array}{c}\text { Low PEEP group } \\
(n=45)\end{array}$ & $\begin{array}{c}\text { High PEEP group } \\
(n=41)\end{array}$ & $P$-value \\
\hline Duration of surgery [min] & $188 \pm 78$ & $218 \pm 105$ & 0.35 \\
\hline PRBCs & $7 \pm 10$ & $7 \pm 8$ & 0.35 \\
\hline FFP & $5 \pm 8$ & $5 \pm 6$ & 0.27 \\
\hline Crystalloids [ml] & $1699 \pm 1126$ & $2233 \pm 1558$ & 0.12 \\
\hline HES [ml] & $420 \pm 417$ & $647 \pm 617$ & 0.11 \\
\hline Gelantine [ml] & $409 \pm 684$ & $450 \pm 783$ & 0.98 \\
\hline TIVA (y/n) & $5 / 25$ & $3 / 23$ & 0.59 \\
\hline Vasopressors (\%) & 90.7 & 97.6 & $N S$ \\
\hline V $_{\mathrm{T}}[\mathrm{ml}]$ & $584.2 \pm 94.8$ & $573 \pm 92.7$ & 0.41 \\
\hline V $_{\mathrm{T}} /$ PBW $[\mathrm{ml} / \mathrm{kg}]$ & $8.4 \pm 1.2$ & $7.8 \pm 1.2$ & 0.014 \\
\hline RR [l/min] & $13.6 \pm 2.5$ & $15.7 \pm 3.2$ & 0.002 \\
\hline PIP [mbar] & $20.2 \pm 5.3$ & $24.3 \pm 3.9$ & $<0.001$ \\
\hline PEEP [mbar] & $5.1 \pm 1.1$ & $10.4 \pm 1.9$ & $<0.001$ \\
\hline FiO & $0.63 \pm 0.23$ & $0.6 \pm 0.26$ & 0.44 \\
\hline
\end{tabular}

PRBC - packed red blood cells, FFP - fresh frozen plasma, HES - hydroxyethyl starch, TIVA - total intravenous anaesthesia, $V_{T}-$ tidal volume, $P B W$ - predicted body weight, $R R$ - respiratory rate, $P I P$ - peak inspiratory pressure, $P E E P$ - positive end-expiratory pressure, FiO, - fraction of inspired oxygen, low PEEP group: < 8 mbar, high PEEP group: $\geq 8$ mbar.

Table III. Mechanical ventilation during ICU stay (median data from POD 1, 3 and 5)

\begin{tabular}{|lccc|}
\hline Parameter & $\begin{array}{c}\text { Low PEEP group } \\
(n=45)\end{array}$ & $\begin{array}{c}\text { High PEEP group } \\
(n=41)\end{array}$ & $P$-value \\
\hline $\mathrm{V}_{\mathrm{T}}[\mathrm{ml}]$ & $624.3 \pm 113.1$ & $667.7 \pm 112.8$ & 0.16 \\
\hline $\mathrm{V}_{\mathrm{T}} / \mathrm{PBW}[\mathrm{ml} / \mathrm{kg}]$ & $9.1 \pm 1.9$ & $9.1 \pm 1.5$ & 0.96 \\
\hline RR $[1 / \mathrm{min}]$ & $15.1 \pm 2.1$ & $16.4 \pm 3.1$ & 0.11 \\
\hline PIP $[\mathrm{mbar}]$ & $22.8 \pm 4.7$ & $25.3 \pm 3.3$ & 0.002 \\
\hline PEEP $[\mathrm{mbar}]$ & $8.7 \pm 2.2$ & $9.2 \pm 2.1$ & 0.004 \\
\hline FiO $_{2}$ & $0.35 \pm 0.10$ & $0.35 \pm 0.08$ & 0.88 \\
\hline
\end{tabular}

Data expressed as mean $\pm S D$. POD - postoperative day, $V_{T}$ - tidal volume, $P B W$ - predicted body weight, $R R$ - respiratory rate, $P I P-p e a k$ inspiratory pressure, PEEP - positive end-expiratory pressure, FiO ${ }_{2}$ - fraction of inspired oxygen; low PEEP group: 8 mbar, high PEEP group: $\geq 8$ mbar. 
Table IV. Outcome parameters

\begin{tabular}{|c|c|c|c|}
\hline Parameter & $\begin{array}{l}\text { Low PEEP group } \\
\qquad(n=45)\end{array}$ & $\begin{array}{l}\text { High PEEP group } \\
\quad(n=41)\end{array}$ & $P$-value \\
\hline Pneumonia (\% within 7 days) & 26.7 & 7.3 & 0.02 \\
\hline \multicolumn{4}{|l|}{ mCPIS (AU): } \\
\hline POD 1 & $2 \pm 2$ & $1 \pm 1$ & 0.44 \\
\hline POD 3 & $3 \pm 2$ & $1 \pm 1$ & $<0.001$ \\
\hline POD 5 & $3 \pm 2$ & $2 \pm 1$ & 0.006 \\
\hline \multicolumn{4}{|l|}{ PF ratio [mm Hg]: } \\
\hline Postoperative & $345 \pm 124$ & $414 \pm 136$ & 0.02 \\
\hline POD 1 & $342 \pm 108$ & $361 \pm 103$ & 0.49 \\
\hline POD 3 & $367 \pm 128$ & $360 \pm 115$ & 0.87 \\
\hline POD 5 & $343 \pm 110$ & $360 \pm 87$ & 0.37 \\
\hline \multicolumn{4}{|l|}{ Lactate $[\mathrm{mmol} / \mathrm{l}]$ : } \\
\hline Postoperative & $3.4 \pm 2.4$ & $3.3 \pm 2.1$ & 0.67 \\
\hline POD 1 & $2.2 \pm 1.8$ & $2.3 \pm 2.1$ & 0.95 \\
\hline POD 3 & $1.1 \pm 0.7$ & $1.2 \pm 1$ & 0.88 \\
\hline POD 5 & $0.9 \pm 0.3$ & $0.9 \pm 0.5$ & 0.61 \\
\hline \multicolumn{4}{|l|}{ Creatinine $[\mu \mathrm{mol} / \mathrm{l}]:$} \\
\hline POD 1 & $93.1 \pm 32.6$ & $97.3 \pm 53.4$ & 0.49 \\
\hline POD 3 & $86.9 \pm 43.6$ & $96.4 \pm 66.0$ & 0.87 \\
\hline POD 5 & $73.9 \pm 27.9$ & $95.6 \pm 77.9$ & 0.22 \\
\hline ARDS (\%) & 22.2 & 24.4 & 0.87 \\
\hline Mortality (\%) & 6.7 & 7.3 & 0.22 \\
\hline
\end{tabular}

emergency surgery. Furthermore, the overall incidence of clinically diagnosed pneumonia as well as mCPIS scores on POD 3 and 5 was significantly lower in this group. There was no difference in the incidence of acute respiratory distress syndrome (ARDS) and mortality up until discharge between groups. There was a significant difference in body mass index (BMI) and in baseline oxygenation between groups. We found no further differences in baseline data, including known prehospital and clinical risk factors for pulmonary complications and the Lung Organ Failure Score. Severity of injury was high in both groups as indicated by a median ISS above 25 , and both groups had a mean AIS for the chest region of 3 , indicating severe chest injury. Thus, both groups were at significant risk of severe pulmonary organ dysfunction with an estimated rate around $15 \%$ as predicted by median LOFS of 16 and 17 points, respectively. There was a statistically significant difference in intraoperative tidal volume and respiratory rate between groups. But the clinical significance of the rather small difference between groups with a tidal volume of $8.4 \pm 1.2 \mathrm{ml}$ in the low PEEP group and 7.8 $\pm 0.9 \mathrm{ml}$ in the high PEEP cannot be determined. Although even small increases of tidal volume beyond $6 \mathrm{ml}$ may be associated with an increased risk of mortality in patients with ARDS [14], the optimal size of intraoperative tidal volume is currently a matter of debate. Most expert reviews suggest an intraoperative tidal volume of approximately 6-8 $\mathrm{ml} / \mathrm{kg}$ [15-17], but in contrast to that a large data analysis showed the lowest mortality in patients ventilated with $8-10 \mathrm{ml} / \mathrm{kg}$ [9]. Incidences of pneumonia, ARDS and mortality in our study were comparable to those reported in the literature [18]. There were no differences in intraoperative vasopressor use, perioperative blood pressures, lactate levels or postoperative creatinine levels before or after surgery between groups.

The rationale for ventilation with moderate or higher PEEP levels is to keep open recruited lung 
areas, improve perioperative oxygenation, limit cyclic opening and closing of dependent lung areas and possibly prevent pleural effusions [19]. On the other hand, some authors propose ventilation with minimal or even no PEEP especially in haemodynamically unstable patients to prevent further impairment of circulation and to limit alveolar overdistension. Cut-off values for low and high PEEP values are not well defined in the literature, and values vary between studies. We predetermined a cut-off value of 8 mbar before data extraction, comparable to a multifaceted bundle strategy for perioperative ventilation published by Futier et al. [15]. Several strategies for setting of perioperative PEEP either derived from measurements of pulmonary mechanics, patient-specific oxygenation or independent from patient-specific measurements have been discussed in the literature. Due to the retrospective design of this study, the rationale for the different PEEP levels used in the studied patients cannot be determined. However, PEEP groups in our study had a significant difference with regards to body weight and preoperative oxygenation. Higher body weight might have led to the lower preoperative oxygenation values (as indicated by PF ratio), which were, although significantly different, still higher than $300 \mathrm{~mm} \mathrm{Hg}$ in both groups. Both higher BMI and lower baseline oxygenation may have caused the different PEEP selection. However, overweight is not only a risk factor for cardiovascular disease [20] but also an independent risk factor for perioperative pneumonia, possibly by increased lung derecruitment, in polytrauma and non-trauma patients [21, 22]. It is also associated with an increased risk for single as well as multiple organ dysfunction and mortality after trauma [23, 24]. Despite those increased risks associated with overweight and obesity, in our study the group with higher PEEP (and also the higher BMI) had a significantly lower incidence of pneumonia and better oxygenation.

Oxygenation as indicated by PF ratio is a frequently used surrogate parameter for pulmonary outcome and lung recruitment in experimental and clinical studies. Nonetheless, several studies have raised concern that an improvement in oxygenation does not positively correlate with an improved outcome [25]. Furthermore, data with respect to the association of high PEEP ventilation and oxygenation are contradictory. While some authors found improved oxygenation and ventilation distribution with higher PEEP, other studies suggested no change in oxygenation and an increase of lung inflammation possibly associated with pulmonary complications [26, 27]. It has been shown in CT studies of ARDS patients that oxygenation correlates well with the area of open lung [28]. In our study, higher PEEP levels were associated with better postoperative oxygenation despite lower baseline oxygenation before surgery. Although the higher PEEP group might need higher PEEP levels to keep the lung recruited because of higher body weight and possibly stiffer chest walls, the difference in postoperative oxygenation suggests better lung recruitment in patients with higher PEEP levels after surgery.

Lung derecruitment is not only associated with ventilator induced lung injury, but atelectasis and inactivation of surfactant are also associated with increased risk of pneumonia, which frequently occurs in multiple trauma patients. Diagnosis is often difficult due to a systemic inflammatory response syndrome (SIRS) and lung contusion after severe trauma. We used a common clinical definition of pneumonia and calculated a modified CPI score (mCPIS). The original score, as described by Pugin et al. [12] and often used in pulmonary research, was found to be imprecise in differentiating systemic inflammatory response syndrome in trauma patients from pneumonia [29]. The modified score we used in this study was validated in patients with traumatic brain injury, although the same limitations may exist in multiple trauma patients [13]. Results in the literature are conflicting with regards to the value of higher PEEP levels in prevention of pneumonia. However, a study using PEEP in postoperative ventilation showed a reduction in ventilator-associated pneumonia (VAP) incidence comparable in size to our study [30]. Moreover, a recent randomized controlled trial demonstrated reduction in the incidence of pneumonia after elective surgery using protective intraoperative ventilation with high PEEP, but it was unclear whether this effect was due to low tidal volume or the higher PEEP of 10 mbar [6]. Several authors have suggested that a lower rate of microaspiration due to higher PEEP levels could reduce VAP incidence when used during intensive care ventilation. In our study, both groups were ventilated with higher PEEP levels postoperatively (> 7 mbar). This fact suggests that other mechanisms may play an important role in prevention of VAP. In a recently published analysis of ventilatory data during general surgery of almost 30000 patients, the use of low PEEP was associated with increased mortality despite protective tidal volumes [9]. These data may suggest that low tidal volume ventilation with inadequate low PEEP increases atelectrauma and may contribute to lung injury. Adversely, the previously published PROVHILO study proposed a low tidal volume ventilation with low PEEP during general anaesthesia for open abdominal surgery [31]. High PEEP levels of 12 mbar were associated with haemodynamic compromise and increased vasopressor use without a difference in postoperative pulmonary 
complications. In our study, higher PEEP was not associated with increased use of intraoperative vasopressors or fluids. Furthermore, our patients had severe thoracic trauma, and thus may have a benefit from high PEEP. There was no difference in postoperative lactate levels, which indicates no differences in organ perfusion between groups.

We found no difference in the incidence of ARDS between groups. Remarkably, in our study both PEEP groups had higher tidal volumes intraoperatively and both groups had a high incidence of chest trauma with a median AIS for the chest region of 3 . These facts and the small sample size may have superimposed preventive effects of PEEP on incidence of ARDS.

Important limitations of this study are due to the retrospective design of our analysis. At first, there is no feasible evidence why the various PEEP levels were chosen by the anaesthetist in the theatre. Although we assessed several known pulmonary risk factors, confounding by unknown factors is possible. To minimize the risk of confounding, we calculated for all patients the LOFS, which is a pulmonary risk predicting score focusing on multiple trauma patients. Moreover, all studied patients were treated in a small specialized ICU with 8 beds, leading to standardised postoperative trauma care. Nevertheless, the results of this study are difficult to generalise due to the highly selected study population. Strict inclusion criteria selected only those patients with the highest risk of pulmonary complications in the early stage of injury. We believe that only interventions in the early stage of injury are suitable for reduction of pulmonary complications. Furthermore, the signal of harm may be difficult to detect due to the small sample size.

In conclusion, higher intraoperative PEEP levels were associated with better oxygenation and reduced incidence of pulmonary complications after major trauma surgery in polytraumatized patients with severe chest injury without impairment of haemodynamic parameters. However, the mechanisms of protection remain unclear, and further experimental and randomized controlled trials should elucidate mechanisms and the influence of PEEP ventilation on outcome after multiple trauma.

\section{Conflict of interest}

The authors declare no conflict of interest.

\section{References}

1. Bhalla K, Harrison JE, Fingerhut LA, Shahraz S, Abraham J, Yeh PH; Global Burden Of Disease Injury Expert Group. The global injury mortality data collection of the Global Burden of Disease Injury Expert Group: a publicly accessible research tool. Int J Inj Contr Saf Promot 2011; 18: 249-53.
2. Minei JP, Cuschieri J, Sperry J, et al. The changing pattern and implications of multiple organ failure after blunt injury with hemorrhagic shock. Crit Care Med 2012; 40: 1129-35.

3. Geiger EV, Lustenberger T, Wutzler S, et al. Predictors of pulmonary failure following severe trauma: a trauma registry-based analysis. Scand I Trauma Resusc Emerg Med 2013; 21: 34.

4. Wutzler S, Wafaisade A, Maegele M, et al. Lung Organ Failure Score (LOFS): probability of severe pulmonary organ failure after multiple injuries including chest trauma. Injury 2012; 43: 1507-12.

5. Ahmed AH, Litell JM, Malinchoc M, et al. The role of potentially preventable hospital exposures in the development of acute respiratory distress syndrome: a population-based study. Crit Care Med 2014; 42: 31-9.

6. Futier E, Constantin JM, Paugam-Burtz C, et al. A trial of intraoperative low-tidal-volume ventilation in abdominal surgery. N Engl J Med 2013; 369: 428-37.

7. Severgnini P, Selmo G, Lanza C, et al. Protective mechanical ventilation during general anesthesia for open abdominal surgery improves postoperative pulmonary function. Anesthesiology 2013; 118: 1307-21.

8. Slutsky AS, Ranieri VM. Ventilator-induced lung injury. N Engl J Med 2013; 369: 2126-36.

9. Levin MA, McCormick PJ, Lin HM, Hosseinian L, Fischer GW. Low intraoperative tidal volume ventilation with minimal PEEP is associated with increased mortality. Br J Anaesth 2014; 113: 97-108.

10. Ambrosio AM, Luo R, Fantoni DT, et al. Effects of positive end-expiratory pressure titration and recruitment maneuver on lung inflammation and hyperinflation in experimental acid aspiration-induced lung injury. Anesthesiology 2012; 117: 1322-34.

11. Johanson WG, Pierce AK, Sanford JP, Thomas GD. Nosocomial respiratory infections with gram-negative bacilli. The significance of colonization of the respiratory tract. Ann Intern Med 1972; 77: 701-6.

12. Pugin J, Auckenthaler R, Mili N, Janssens JP, Lew PD, Suter PM. Diagnosis of ventilator-associated pneumonia by bacteriologic analysis of bronchoscopic and nonbronchoscopic "blind" bronchoalveolar lavage fluid. Am Rev Respir Dis 1991; 143: 1121-9.

13. Pelosi P, Barassi A, Severgnini P, et al. Prognostic role of clinical and laboratory criteria to identify early ventilator-associated pneumonia in brain injury. Chest 2008; 134: 101-8.

14. Needham DM, Yang T, Dinglas VD, et al. Timing of low tidal volume ventilation and ICU mortality in ARDS: a prospective cohort study. Am J Respir Crit Care Med 2014; 191: 177-85.

15. Futier E, Marret E, Jaber S. Perioperative positive pressure ventilation: an integrated approach to improve pulmonary care. Anesthesiology 2014; 121: 400-8.

16. Goldenberg NM, Steinberg BE, Lee WL, Wijeysundera DN, Kavanagh BP. Lung-protective ventilation in the operating room: time to implement? Anesthesiology 2014; 121: 184-8.

17. Serpa Neto A, Simonis FD, Schultz MJ. How to ventilate patients without acute respiratory distress syndrome? Curr Opin Crit Care 2015; 21: 65-73.

18. Benns M, Carr B, Kallan MJ, Sims CA. Benchmarking the incidence of organ failure after injury at trauma centers and nontrauma centers in the United States. J Trauma Acute Care Surg 2013; 75: 426-31.

19. Yildiz Y, Salihoglu E, Celik S, et al. The effect of postoperative positive end-expiratory pressure on postoperative 
bleeding after off-pump coronary artery bypass grafting. Arch Med Sci 2014; 10: 933-40.

20. Zhu J, Su X, Li G, Chen J, Tang B, Yang Y. The incidence of acute myocardial infarction in relation to overweight and obesity: a meta-analysis. Arch Med Sci 2014; 10 : 855-62.

21. Nie W, Zhang Y, Jee SH, Jung KJ, Li B, Xiu Q. Obesity survival paradox in pneumonia: a meta-analysis. BMC Med 2014; 12: 61.

22. Mica L, Keller C, Vomela J, Trentz O, Plecko M, Keel MJ. Obesity and overweight as a risk factor for pneumonia in polytrauma patients: a retrospective cohort study. J Trauma Acute Care Surg 2013; 75: 693-8.

23. Ciesla DJ, Moore EE, Johnson JL, Burch JM, Cothren CC, Sauaia A. Obesity increases risk of organ failure after severe trauma. J Am Coll Surg 2006; 203: 539-45.

24. Neville AL, Brown CVR, Weng J, Demetriades D, Velmahos GC. Obesity is an independent risk factor of mortality in severely injured blunt trauma patients. Arch Surg 2004; 139: 983-7.

25. Adhikari NKJ, Burns KEA, Friedrich JO, Granton JT, Cook DJ, Meade MO. Effect of nitric oxide on oxygenation and mortality in acute lung injury: systematic review and meta-analysis. BMJ 2007; 334: 779.

26. Karsten J, Heinze H, Meier T. Impact of PEEP during laparoscopic surgery on early postoperative ventilation distribution visualized by electrical impedance tomography. Minerva Anestesiol 2014; 80: 158-66.

27. Borges DL, Nina VJ da S, Costa M de AG, et al. Effects of different PEEP levels on respiratory mechanics and oxygenation after coronary artery bypass grafting. Rev Bras Cir Cardiovasc 2013; 28: 380-5.

28. Borges JB, Okamoto VN, Matos GFJ, et al. Reversibility of lung collapse and hypoxemia in early acute respiratory distress syndrome. Am J Respir Crit Care Med 2006; 174: 268-78.

29. Croce MA, Swanson JM, Magnotti LJ, et al. The futility of the clinical pulmonary infection score in trauma patients. J Trauma 2006; 60: 523-7.

30. Manzano F, Fernández-Mondéjar E, Colmenero M, et al. Positive-end expiratory pressure reduces incidence of ventilator-associated pneumonia in nonhypoxemic patients. Crit Care Med 2008; 36: 2225-31.

31. Hemmes SNT, Gama de Abreu M, Pelosi P, Schultz MJ. High versus low positive end-expiratory pressure during general anaesthesia for open abdominal surgery (PROVHILO trial): a multicentre randomised controlled trial. Lancet 2014; 384: 495-503. 